Rojo-Ramos, J., Ferrera-Granados, C., Mañanas-Iglesias, C. \& Guevara-Pérez, J.C. (2022). Estudio descriptivo de Ciber victimización en una muestra de estudiantes de Educación Secundaria Obligatoria. Revista Electrónica Interuniversitaria de Formación del Profesorado, 25(1), 117-130.

DOI: https://doi.org/10.6018/reifop.508151

\title{
Estudio descriptivo de Cibervictimización en una muestra de estudiantes de Educación Secundaria Obligatoria
}

Jorge Rojo Ramos ${ }^{(1)}$, Carlos Ferrera Granados ${ }^{(1)}$, Carlos Mañanas Iglesias ${ }^{(1)}$, Juan Carlos Guevara Pérez ${ }^{(2)}$

(1) Universidad de Extremadura, ${ }^{(2)}$ Universidad de Zaragoza

\section{Resumen}

Durante la adolescencia se producen los mayores riesgos de un mal uso de las nuevas tecnologías, y con ello, la proliferación del ciberacoso. El objetivo del presente trabajo es realizar un análisis descriptivo de cibervictimización en función del género y curso en una muestra de estudiantes de Educación Secundaria Obligatoria de un colegio concertado en la ciudad de Cáceres. La metodología empleada en la investigación es básica, no experimental, descriptiva-correlacional. Participaron un total de 141 alumnos pertenecientes a cursos de toda la etapa de la ESO. Los resultados evidencian una elevada digitalización de los hogares (99,3\%). También se desprende que la cibervictimización verbal-escrita es la más común en los adolescentes para llevar a cabo el ciberacoso.

\section{Palabras clave}

Adolescencia; Nuevas Tecnologías; Ciberacoso; Género

\section{Contacto:}

Jorge Rojo-Ramos, jorgerr@unex.es, University of Extremadura, 10003 Cáceres, Spain. 


\title{
Descriptive study of Cybervictimization in a sample of Compulsory Secondary Education students
}

\begin{abstract}
During adolescence, the greatest risks of misuse of new technologies occur, and with it, the proliferation of cyberbullying. The objective of this work is to carry out a descriptive analysis of cybervictimization according to gender and grade in a sample of Compulsory Secondary Education students from a private school in the city of Cáceres. The methodology used in the research is basic, non-experimental, descriptive-correlational. A total of 141 students from courses throughout the ESO stage participated. The results show a high digitization of households (99.3\%). It also appears that verbal-written cybervictimization is the most common in adolescents to carry out cyberbullying.
\end{abstract}

\section{Keywords}

Adolescence; New technologies; Cyber bullying; Gender.

\section{Introducción}

En la actualidad, los aportes de la inclusión de las nuevas tecnologías en el ámbito de la educación resultan incuestionables. No obstante, dichas tecnologías se han convertido en instrumentos susceptibles de generar daño considerable a terceros sin el adecuado control de sus padres o tutores legales (Amemiya, I., Oliveros, M., \& Barrientos, A., 2009).

Debido a la gran acogida de los ordenadores y smartphones entre los adolescentes, su uso incorrecto está a la orden del día, siendo las herramientas a través de las cuales se lleva a cabo el ciberacoso. Esta problemática está pasando rápidamente de la universidad a niveles inferiores como el de la Educación Secundaria Garaigordobil y Oñederra, (2010), siendo muy preocupante a tan temprana edad, no solo por la violencia que pueda originar entre pares, sino por la posibilidad de agresiones de índole sexual ocasionada por mayores como el Grooming.

Un reporte de UNICEF (2019) señala que en cada aula existen aproximadamente dos alumnos que sufren ciberbullying. Su rápido crecimiento viene facilitado por la creciente accesibilidad al uso de nuevas tecnologías por parte de los adolescentes. Adiconalmente, la falta de formación del profesorado para su temprana detección, y la escasa resistencia de las víctimas ante los ciberataques incrementa las consecuencias negativas para las víctimas. Existen estudios que relacionan la incidencia del ciberbullying con el rendimiento académico (Avilés, 2009; García y Jiménez, 2010).

Por todo ello la presente investigación tiene como objetivo realizar un análisis descriptivo de cibervictimización en una muestra seleccionada de estudiantes de la etapa educativa de la Educación Secundaria Obligatoria en función del género y curso. Para ello, después de esta introducción un análisis de la literatura aporta el estado actual de la cuestión y el marco teórico de referencia a partir del cual se llevó a cabo la investigación. Seguidamente se presentan las hipótesis de la investigación, desarrollando la metodología a implementar. Posteriormente se analizan y discuten los resultados contrastándolos con la literatura y finalmente se exponen las conclusiones obtenidas con las limitaciones del estudio y futuras líneas de trabajo. 


\section{Literatura previa}

\section{Estado de la cuestión}

El peligro asociado al uso indiscriminado que realizan los jóvenes de las nuevas tecnologías, y de cómo dichas tecnologías e Internet se están convirtiendo en la actualidad en uno de los medios más comunes para delinquir, dada la importancia de internet como herramienta para la comunicación entre iguales (Sapountzi y Psannis, 2018).

Son las redes sociales (RRSS) como WhatsApp, Instagram, Youtube o Facebook las favoritas por los jóvenes españoles, y el teléfono móvil la principal fuente de acceso para navegar por internet con un $95 \%$ de prevalencia. A este respecto, al ser los más jóvenes los consumidores más activos, se convierten en los más vulnerables a los riesgos asociados a un mal uso de las tecnologías (labSpain, 2019).

Debido a que la mayor parte de los adolescentes en la actualidad hacen un uso constante de las RRSS y de internet (Martín, A., Cabré, R., \& Neri, E., 2019), los niveles de ciberacoso durante la pandemia acaecida por la COVID-19, se han incrementado de forma exponencial entre los más jóvenes al disponer de una mayor cantidad de tiempo frente a una pantalla.

Según Blanco, González y Velasco (2020) son los adolescentes en las etapas de la Educación Secundaria Obligatoria y Universitaria quienes más usan las tecnologías para socializar. Esta nueva forma de comunicarse ha generado un estilo de convivencia donde cada adolescente forma su identidad digital, revelando en muchas ocasiones algunos datos personales que pueden hacerles ser víctimas de los ciberdelincuentes que navegan por la red (López y Sánchez, 2019).

Entidades como la Royal Society for Public Health (2017), muestra preocupación ante la salud mental de los adolescentes y el uso inadecuado de las RRSS. En esta línea, el fenómeno del ciberacoso ha generado una nueva tipología de víctimas, que puede verse seriamente afectadas a nivel psicológico (Wolke, D., \& Lereya, S. T. (2015).

El ciberacoso es considerado por la Organización Mundial de la Salud como un grave problema de salud pública que afecta de manera negativa y exponencial la salud física y mental de los adolescentes, tanto cibervíctimas como ciberagresores, estando íntimamente ligado al desajuste social y psicológico en edades tempranas (Garaigordobil y Oñederra, 2010).

Pero no todo es negativo. La literatura también documenta numerosas iniciativas y experiencias positivas (Del Rey et al., 2018).

\section{Conceptualización}

Entendemos bullying como "aquel comportamiento prolongado de insulto verbal, rechazo social, intimidación psicológica y/o agresión física de uno o un grupo de individuos contra otro, el cual se convierte en víctima" (Espelage y Swearer, 2003). Willard, (2006) definen el cyberbullyn como "el envío y acción de colgar (sending y posting) textos, imágenes dañinas o crueles por internet u otros medios digitales de comunicación", y Belsey (2005) como "aquel uso vejatorio de algunas tecnologías de la información y la comunicación por parte de un individuo o grupo que de manera deliberada, repetitiva y hostil pretende dañar a una persona". A modo general, bullying y ciberbullying son fenómenos muy parejos, ya que en ambos existe una intencionalidad clara de dañar y vejar a un tercero, pero la metodología y el contexto donde florece cada uno de ellos es diferente.

Kowalski et al., (2010) destacan los insultos electrónicos (flame war) entre los diferentes métodos de acoso llevados a cabo por los ciberacosadores. Otro método es el 
sonsacamiento, donde el acosador se acerca a la víctima de manera amigable con el fin de establecer una relación de confianza para que la víctima le comparta información confidencial que luego será desvelada y publicada por este fake friend facilitando que la víctima sea objeto de ciberacoso, chantaje y humillación. La ciberpersecución o "cyberstalking" está también muy ligada al hostigamiento, y consiste en el envío masivo de mensajes amenazantes, coacciones y demás insultos de manera consecutiva, generando en la víctima sentimientos de indefensión y temor incluso por su propia integridad física.

Giménez Gualdo et al., (2013) señala algunos roles que podemos encontrar en una situación de ciberacoso. Estos son el ciberbullie o ciberacosador, la cibervíctima que recibe el ciberacoso, y cibertestigo, que son aquellos individuos conocedores de la situación de ciberacoso. Por otra parte, autores como De la Plaza y González, (2019) establecen una clasificación de las víctimas, y una tipología del ciberacosador. Adicionalmente, Fernández et al., (2015) asocia factores de tipo personal, familiar y escolar con el ciberacoso. Baldry y Farrington, (2015) señalan factores de protección o riesgo procedentes de familias. Hernández y O`Reilly (2015) señala también como las tensiones matrimoniales o las situaciones socioeconómicas pueden contribuir al cyberbullyn. Fernández et al., (2015) destaca que puede ser consecuencia de métodos permisivos por parte de los padres. También, Díaz Aguado, (2005) señala a la Escuela como facilitadora de estas conductas.

En cuanto al marco regulador español, los artículos 172 y 173 del Código Penal tipifican la comisión de este delito debe cumplir. Las injurias también son parte de los delitos más asiduos en los casos de ciberbullying. Estas aparecen reguladas en los artículos 206 a 210 del Código Penal. También las calumnias vienen recogidas en el artículo 205 del Código Penal. La edad del sujeto en estos casos será la que establezca la diferencia, y por ende, la regulación penal. En este sentido, en España existen dos leyes que regulan estos casos, la Ley Orgánica 10/1995, de 23 de noviembre, por la que se aprueba el Código Penal y la Ley Orgánica 5/2000, de 12 de enero, reguladora de la responsabilidad penal de los menores (LORPM).

En cuanto a la normativa reguladora de las RRSS la Ley 34/2002, de 11 de julio, de Servicios de la Sociedad de la Información y de Comercio Electrónico (LSSI) regula las RRSS. El Reglamento Europeo de Protección de Datos (RGPD), el tratamiento de los datos en la red, la Ley Orgánica 3/2018 de Protección de Datos Personales y Garantía de los Derechos Digitales (LOPD), garantiza el acceso universal a internet de forma segura e inclusiva.

Para contrastar la hipótesis de si existe diferencia en función del género con respecto a la cibervictimización en estudiantes de secundaria, la presente investigación realiza un análisis descriptivo de cibervictimización en una muestra de estudiantes de la etapa educativa de Educación Secundaria Obligatoria en función del género y curso.

\section{Metodología}

Se desarrolla una metodología de investigación mixta (no experimental, descriptivacorrelacional), con carácter descriptivo trasversal a partir de un cuestionario aplicado sobre una muestra de alumnado de Educación Secundaria Obligatoria.

\section{Participantes}

Han participado un total de 141 alumnos de toda la etapa, desde los 12 a los 16 años de edad, siendo la distribución de 75 alumnas (53,2\%) y 66 alumnos (46,8\%). En el muestreo, todos los participantes fueron informados previamente de los objetivos y el fin de la investigación, así como a sus familias al tratarse de menores de edad (Consentimiento informado - Anexo I). La encuesta ha sido anónima y voluntaria, cumpliendo con la Ley Orgánica de Protección de 
Datos de Carácter Personal 15/99. Los participantes del estudio fueron seleccionados mediante un método de muestreo no probabilístico basado en un muestreo por conveniencia, (Salkind, 1999).

\section{Tabla 1.}

Distribución de frecuencias de la muestra ( $N=141)$.

\begin{tabular}{cccc}
\hline Variable & Categorías & N & $\%$ \\
\hline \multirow{2}{*}{ Sexo } & Hombre & 66 & 46,8 \\
& Mujer & 75 & 53,2 \\
& Primero de la E.S.O. & 43 & 30,5 \\
\multirow{4}{*}{ Curso } & Segundo de la E.S.O. & 41 & 29,1 \\
& Tercero de la E.S.O. & 37 & 26,2 \\
& Cuarto de la E.S.O. & 20 & 14,2 \\
Variable & & $\mathbf{M}$ & $\mathbf{d t}$ \\
\hline Edad & & 13,73 & 1,2 \\
\hline
\end{tabular}

La tabla 1 muestra la distribución de frecuencia de la muestra en cuanto a la variable sexo, curso y edad, con un total de 141 participantes $(\mathrm{N}=141)$ de los que el 46,8\% (66) son hombres frente al 53,2\% (75) de mujeres. Por cursos, la participación en el estudio ha tenido mayor calado en los cursos más bajos de la etapa y por ende los participantes más jóvenes. En este sentido, relativo a $1^{\circ}$ de la ESO representan un total del 30,5\% (43), seguido del $2^{\circ}$ ESO con un $29,1 \%$ (41). $3^{\circ}$ de la ESO es representado por un $26,1 \%$ (37), y $4^{\circ}$ ESO con un $14,2 \%$ (20). La media de edad obtenida por los participantes es de 13,73 años $(\mathrm{dt}=1,2)$.

\section{Instrumentos}

Para la recolección de los datos se elaboró un cuestionario a través del empleo de Google Docs, constituido por 3 preguntas (sexo, curso y edad). Además, se incluyeron posteriormente 8 preguntas sobre aspectos relativos al uso de las nuevas tecnologías en la adolescencia y el fenómeno del ciberacoso.

Adicionalmente, se ha incorporado el cuestionario de Cibervictimización en estudiantes de Secundaria (Álvarez-García et al, 2014) compuesto por 26 ítems agrupados en 4 dimensiones. En cada dimensión se hace referencia a algún aspecto específico tal y como se detalla a continuación: dimensión 1 (cibervictimización visual-4 ítems); dimensión 2: (exclusión online4 ítems); dimensión 3 (suplantación-5 ítems) y dimensión 4: (cibervictimización verbal/escrita12 ítems). En todos ellos se describen diferentes tipos de ciberagresiones realizadas a través de las nuevas tecnologías. En este cuestionario el participante deberá indicar con qué frecuencia ha sido víctima de cada una de las situaciones citadas en el mismo durante los últimos 3 meses, a través de una escala de respuesta tipo Likert con cuatro posibilidades de respuesta, $1=$ nunca, $2=$ pocas veces, $3=$ =muchas veces y $4=$ =siempre. (Anexo I).

Relativo a la confiabilidad del instrumento, se ha obtenido un alfa de Cronbach de 0,85 , lo que atribuye robustez al cuestionario empleado para su aplicación en el contexto de la presente investigación. 


\section{Procedimiento}

Los cuestionarios han sido diseñados y aplicados a través de la herramienta Google Forms. Los e-cuestionarios permiten un mayor ahorro de costes y tiempo, alta precisión, mayor índice de tasas de retorno y una entrega rápida (Anderson y Kanuka, 2002). Todas las respuestas quedaron almacenadas en una hoja de cálculo que facilitó la transformación de los mismos para el análisis estadístico. La recogida de datos se desarrolló durante el curso académico en los meses de abril y mayo de 2021.

Se presentaron los objetivos de la investigación al equipo directivo del colegio Santa Cecilia "Carmelitas" de Cáceres, quienes tras su visto bueno facilitaron la ejecución de la investigación en las aulas de Educación Secundaria Obligatoria. Posterior a ello, se convocó una reunión telemática vía Google Meet para informar a los tutores sobre la investigación de cara a los consentimientos informados de padres/madres/tutores legales de los estudiantes por ser menores de edad. Obtenidos los permisos necesarios, se facilitó el URL a los cuestionarios a través de la plataforma Google Classroom. Todos los datos fueron recogidos de forma anónima. El tiempo medio para responder a los cuestionarios fue de 10 minutos.

\section{Análisis y Discusión de Resultados}

Para el análisis de datos se utilizó el Statistical Package for Social Sciences (SPSS). La prueba de Kolmogorov Smirov indicó que las variables estudiadas no cumplían con el supuesto de normalidad, por lo que se decidió utilizar pruebas no paramétricas. En primer lugar, el test Chi-Cuadrado de Pearson permitió analizar las diferencias obtenidas de los 8 ítems previos al cuestionario de cibervictimización en función al género. Los resultados se presentan en la Tabla 2:

Tabla 2.

Distribución de frecuencias en las respuestas a los ítems según género.

Q1. ¿Has sufrido alguna situación de ciberacoso por parte de algún compañero/a?

\begin{tabular}{|c|c|c|c|c|c|c|}
\hline \multirow{7}{*}{ Sexo } & \multirow{3}{*}{ Hombres } & & $\mathrm{Si}$ & & No & $p$ \\
\hline & & $\mathrm{N}$ & 6 & & 60 & \multirow{6}{*}{0,32} \\
\hline & & $\%$ & 4,3 & & 42,6 & \\
\hline & \multirow{2}{*}{ Mujeres } & $N$ & 10 & \multirow{2}{*}{\multicolumn{2}{|c|}{$\begin{array}{l}65 \\
65\end{array}$}} & \\
\hline & & $\%$ & 7,1 & & & \\
\hline & \multirow{2}{*}{ Total Sexo } & $\mathrm{N}$ & 16 & & & \\
\hline & & $\%$ & 11,3 & \multicolumn{2}{|r|}{88,7} & \\
\hline \multicolumn{7}{|c|}{$\begin{array}{l}\text { Q2. ¿Has sido parte directa de alguna situación de ciberacoso hacia } \\
\text { compañeros de clase? }\end{array}$} \\
\hline \multirow{7}{*}{ Sexo } & \multirow{3}{*}{ Hombres } & & $\mathrm{Si}$ & No & Tal vez & $\mathrm{p}$ \\
\hline & & $\mathrm{N}$ & 1 & 60 & 5 & \multirow{6}{*}{0,81} \\
\hline & & $\%$ & 0,7 & 42,6 & 3,5 & \\
\hline & Muieres & $\mathrm{N}$ & 1 & 66 & 8 & \\
\hline & Iviujeres & $\%$ & 0,7 & 46,8 & 5,7 & \\
\hline & \multirow{2}{*}{ Total Sexo } & $\mathrm{N}$ & 2 & 126 & 13 & \\
\hline & & $\%$ & 1,4 & 89,4 & 9,2 & \\
\hline
\end{tabular}




\begin{tabular}{|c|c|c|c|c|c|}
\hline \multicolumn{6}{|c|}{$\begin{array}{l}\text { Q3. ¿Crees que tu centro educativo lleva a cabo medidas preventivas contra el } \\
\text { fenómeno del ciberacoso? }\end{array}$} \\
\hline \multirow{7}{*}{ Sexo } & \multirow{3}{*}{ Hombres } & & $\mathrm{Si}$ & No & $p$ \\
\hline & & $\mathrm{N}$ & 45 & 21 & \multirow{6}{*}{0,3} \\
\hline & & $\%$ & 31,9 & 14,9 & \\
\hline & \multirow{2}{*}{ Mujeres } & $\mathrm{N}$ & 57 & 18 & \\
\hline & & $\%$ & 40,4 & 12,8 & \\
\hline & \multirow{2}{*}{ Total Sexo } & $\mathrm{N}$ & 102 & 39 & \\
\hline & & $\%$ & 72,3 & 27,7 & \\
\hline \multicolumn{6}{|c|}{ Q4. ¿Tienes internet en casa? } \\
\hline \multirow{7}{*}{ Sexo } & \multirow{3}{*}{ Hombres } & & $\mathrm{Si}$ & No & $p$ \\
\hline & & $\mathrm{N}$ & 66 & 0 & \multirow{6}{*}{0,34} \\
\hline & & $\%$ & 46,8 & 0 & \\
\hline & Muieres & N & 74 & 1 & \\
\hline & \multirow{3}{*}{ Total Sexo } & $\%$ & 52,5 & 0,7 & \\
\hline & & N & 140 & 1 & \\
\hline & & $\%$ & 99,3 & 0,7 & \\
\hline
\end{tabular}

Q5. ¿Crees que a las personas que sufren ciberacoso les influye en su posterior endimiento académico de forma negativa?

\begin{tabular}{|c|c|c|c|c|c|c|}
\hline & & & $\mathrm{Si}$ & No & Tal vez & $p$ \\
\hline \multirow{6}{*}{ Sexo } & \multirow{2}{*}{ Hombres } & $\mathrm{N}$ & 45 & 2 & 19 & \multirow{6}{*}{0,35} \\
\hline & & $\%$ & 31,9 & 1,4 & 13,5 & \\
\hline & Muieres & $\mathrm{N}$ & 59 & 2 & 14 & \\
\hline & \multirow{3}{*}{ Total Sexo } & $\%$ & 51,8 & 1,4 & 9,9 & \\
\hline & & $\mathrm{N}$ & 104 & 4 & 33 & \\
\hline & & $\%$ & 73,8 & 2,8 & 23,4 & \\
\hline \multicolumn{7}{|c|}{ Q6. ¿Crees que existe ciberacoso en tu centro educativo en los cursos de la ESO? } \\
\hline \multirow{7}{*}{ Sexo } & \multirow{3}{*}{ Hombres } & & $\mathrm{Si}$ & No & Lo desconozco & $p$ \\
\hline & & $\mathrm{N}$ & 14 & 15 & 37 & \multirow{6}{*}{$*_{0,02}$} \\
\hline & & $\%$ & 9,9 & 10,6 & 26,2 & \\
\hline & Muieres & $\mathrm{N}$ & 30 & 8 & 37 & \\
\hline & & $\%$ & 21,3 & 5,7 & 26,2 & \\
\hline & \multirow{2}{*}{ Total Sexo } & $\mathrm{N}$ & 44 & 23 & 74 & \\
\hline & & $\%$ & 31,2 & 16,3 & 52,5 & \\
\hline \multicolumn{7}{|c|}{ Q7. ¿Dispones de teléfono móvil? } \\
\hline \multirow{7}{*}{ Sexo } & \multirow{3}{*}{ Hombres } & & $\mathrm{Si}$ & No & Algunas veces & $p$ \\
\hline & & $\mathrm{N}$ & 60 & 4 & 2 & \multirow{6}{*}{0,6} \\
\hline & & $\%$ & 42,6 & 2,8 & 1,4 & \\
\hline & Muieres & $\mathrm{N}$ & 71 & 2 & 2 & \\
\hline & Miviuetes & $\%$ & 50,4 & 1,4 & 1,4 & \\
\hline & \multirow{2}{*}{ Total Sexo } & $\mathrm{N}$ & 131 & 6 & 4 & \\
\hline & & $\%$ & 92,9 & 4,3 & 2,8 & \\
\hline
\end{tabular}

Q8. ¿Dispones de ordenador, tablet u otro dispositivo con conexión a internet?

Sexo

Hombres N

S1

Si
Algunas veces 


\begin{tabular}{ccccc} 
& $\%$ & 43,3 & 0,7 & 2,8 \\
\multirow{5}{*}{ Mujeres } & $\mathrm{N}$ & 74 & 0 & 1 \\
& $\%$ & 52,5 & 0 & 0,7 \\
\multirow{2}{*}{ Total Sexo } & $\mathrm{N}$ & 135 & 1 & 5 \\
& $\%$ & 95,7 & 0,7 & 3,5 \\
\hline
\end{tabular}

En el ítem Q1 88,7\% de los encuestados afirma no haber experimentado situaciones de ciberacoso frente a un 11,3\% que manifiesta haberlas experimentado. En cuanto al género, las diferencias son significativas ya que del $11,4 \%$ del total de víctimas, el 7,1\% se corresponde con chicas frente al $4,3 \%$ en chicos. Estos resultados corroboran la prevalencia del ciberacoso en el género femenino sobre el masculino observada por Ballesteros et al., (2018).

En el otro lado de la balanza encontramos el perfil del ciberacosador. Según los resultados la gran mayoría del alumnado niega su participación directa o indirecta en situaciones de ciberacoso $(89,4 \%)$, frente a los que afirman haberlo hecho $(1,4 \%)$, y un $9,2 \%$ que afirma haber sido cómplices. Aunque el estudio es anónimo, el porcentaje de ciberacosadores puede ser mayor, ya que la mayoría no suele reconocer sus actos, sino que refugian en el anonimato. Con respecto a estudios que afirman que el género masculino es más propenso a desempeñar el rol de ciberacosador, el estudio no encuentra diferencias significativas entre géneros.

Un $72,3 \%$ de los encuestados afirma que el centro adopta medidas durante el curso académico de forma preventiva contra el fenómeno (Q3) frente al 27,7\% que afirma lo contrario. En cuanto a géneros no encontramos diferencias significativas, pero sí una mejor valoración por parte de los chicos (14,9\%) frente a las chicas (12,8\%). Adicionalmente, el $99,3 \%$ de los encuestados en el presente estudio afirman disponer de acceso a internet en sus domicilios (Q4).

También el 73,8\% de los encuestados afirma que el ciberacoso influye negativamente en el rendimiento académico de los alumnos (Q5), tal y como señalan Avilés, (2009) y García y Jiménez, (2010).

Al valorar la prevalencia del ciberacoso en las aulas (Q6), 52,5\% de los encuestados desconoce este hecho, $16,3 \%$ lo niega y $31,2 \%$ afirman que existe. Des estos últimos $21,3 \%$ son chicas y $9,9 \%$ chicos. Los resultados corroboran el inicio precoz de la violencia cibernética asociado a la adolescencia alertado por Garaigordobil y Oñederra, (2010).

Con respecto a la disponibilidad de dispositivos móviles (Q7), 92,9\% de los encuestados respondió tenerlos, $4,3 \%$ que no y $2,8 \%$ tenerlo ocasionalmente, pero no de manera constante. No se observaron diferencias significativas entre géneros. A este respecto, Sapountzi y Psannis, (2018) advierten sobre el teléfono móvil como medio favorito por los ciberacosadores para cometer sus delitos. Finalmente, el $95,7 \%$ posee dispositivos electrónicos con acceso a internet de manera continuada (Q8). A este respecto, Flores J., (2008) señala que la digitalización puede traer en la misma proporción un mal uso de las nuevas tecnologías, desencadenando en fraudes, adicciones, aparición de ciberdelitos (grooming, sexting, ciberbullying), y problemas de seguridad.

Para al análisis descriptivo de los ítems del cuestionario de cibervictimización y sus posibles diferencias en términos de género, la prueba $U$ de Mann-Whitney se ha empleado para analizar las relaciones entre los ítems de las diferentes dimensiones en función del género (tablas 3 y 4 ). 


\section{Tabla 3.}

Análisis descriptivo y diferencias por género de los ítems del cuestionario.

\begin{tabular}{|c|c|c|c|c|}
\hline \multirow[b]{2}{*}{ Ítem } & \multicolumn{4}{|c|}{ Género } \\
\hline & $\begin{array}{c}\text { Total } \\
\text { Me(IQR) }\end{array}$ & $\begin{array}{l}\text { Mujer } \\
\text { Me(IQR) }\end{array}$ & $\begin{array}{l}\text { Hombre } \\
\mathrm{Me}(\mathrm{IQR})\end{array}$ & $p$ \\
\hline $\begin{array}{l}\text { 1. Se han hecho pasar por mí en Internet } \\
\text { publicando comentarios a mi nombre, como si } \\
\text { fuese yo. }\end{array}$ & $1(0)$ & $1(0)$ & $1(0)$ & 0,86 \\
\hline $\begin{array}{l}\text { 2. Han copiado conversaciones privadas mías y } \\
\text { se las han enviado a otros, para dañarme. }\end{array}$ & $1(1)$ & $1(1)$ & $1(0)$ & $*_{0,02}$ \\
\hline $\begin{array}{l}\text { 3. Alguna persona no me ha admitido o me ha } \\
\text { expulsado de su equipo en juegos online, sin } \\
\text { haber hecho yo nada malo que lo justifique. }\end{array}$ & $1(1)$ & $1(1)$ & $1(1)$ & 0,41 \\
\hline $\begin{array}{l}\text { 4. Han colgado en Internet fotos mías trucadas } \\
\text { (modificadas), para hacerme daño o reírse de } \\
\text { mí. }\end{array}$ & $1(0)$ & $1(0)$ & $1(0)$ & 0,44 \\
\hline $\begin{array}{l}\text { 5. Alguien se ha hecho pasar por otra persona, } \\
\text { para reírse de mí a través de Internet o del } \\
\text { teléfono móvil. }\end{array}$ & $1(0)$ & $1(0)$ & $1(0)$ & 0,12 \\
\hline $\begin{array}{l}\text { 6. Me han echado o no me han aceptado en la } \\
\text { lista de contactos de algún chat, red social (por } \\
\text { ejemplo, Instagram) o programa de mensajería } \\
\text { instantánea (por ejemplo, Messenger o } \\
\text { WhatsApp) sin haber hecho nada, solo por ser } \\
\text { yo. }\end{array}$ & $1(1)$ & $1(1)$ & $1(1)$ & 0,36 \\
\hline $\begin{array}{l}\text { 7. Me han enviado fotos o vídeos "fuertes" } \\
\text { desagradables para mí. }\end{array}$ & $1(0)$ & $1(0)$ & $10)$ & 0,6 \\
\hline $\begin{array}{l}\text { 8. He recibido llamadas a mi teléfono móvil, } \\
\text { que no contestan, con afán de fastidiar. }\end{array}$ & $1(0)$ & $1(1)$ & $1(1)$ & 0,08 \\
\hline $\begin{array}{l}\text { 9. Han colgado en Internet fotos o vídeos míos } \\
\text { reales comprometidos, sin mi permiso, para } \\
\text { hacerme daño o reírse de mí. }\end{array}$ & $1(0)$ & $1(0)$ & $1(0)$ & 0,9 \\
\hline $\begin{array}{l}\text { 10. Se han publicado en Internet informaciones } \\
\text { que había dado en secreto a un "amigo/a", } \\
\text { esperando que no se lo dijesen a nadie y que } \\
\text { ahora me dañan. }\end{array}$ & $1(0)$ & $1(0)$ & $1(0)$ & 0,53 \\
\hline $\begin{array}{l}\text { 11. He recibido llamadas de amigos y/o } \\
\text { compañeros/as para insultarme o burlarse de } \\
\text { mí. }\end{array}$ & $1(0)$ & $1(0)$ & $1(0)$ & 0,25 \\
\hline $\begin{array}{l}\text { 12. Me han bloqueado el acceso al correo } \\
\text { electrónico, a una red social (Instagram, } \\
\text { Snapchat) o a un programa de mensajería } \\
\text { instantánea (WhatsApp) cambiando mi } \\
\text { contraseña. }\end{array}$ & $1(0)$ & $1(0)$ & $1(0)$ & 0,13 \\
\hline
\end{tabular}


13. Compañeros/as y/o amigos/as se han burlado de mí con comentarios ofensivos e insultantes en las RRSS.

$1(0)$

14. Me han agredido físicamente, lo han grabado y luego lo han difundido.

15. He recibido insultos a través de mensajes cortos de texto (SMS) o programas de mensajería instantánea (WhatsApp).

16. Se han hecho pasar por mí en Instagram, Tik Tok, Snapchat, Facebook, Twitter...creando un falso perfil de usuario (foto, datos personales) con el que me han insultado o ridiculizado.

17. He recibido mensajes anónimos (correos electrónicos, WhatsApp, SMS...) en los que se me amenazaba o atemorizaba.

18. Se han hecho quejas falsas sobre mí en algún foro, red social o juego online, que han hecho que me expulsen del sitio web.

19. Han reenviado a otras personas, para dañarme, correos o mensajes privados que yo había enviado.

20. Me han obligado a hacer algo humillante, lo han grabado y luego lo han difundido para burlarse de mí.

21. Me han amenazado públicamente a través de las RRSS (Facebook, Twitter, Instagram, Telegram, SnapChat...)

22. Amigos/as y/o compañeros/as se ponen de acuerdo para hacerme el vacío (ignorarme) en las RRSS.

23. He recibido llamadas anónimas para amenazarme $\mathrm{o}$ atemorizarme.

24. He recibido comentarios de carácter sexual no deseados a través de Internet.

25. Alguien que ha conseguido mi contraseña ha enviado mensajes molestos a algún conocido/a, como si hubiese sido yo, para meterme en líos.

26. Se han publicado rumores falsos sobre mí en alguna red social.
$1(0)$

$1(0) \quad 1(0) \quad 0,66$

$1(0) \quad 1(0) \quad 0,92$

$1(1) \quad 1(1) \quad 1(1) \quad 0,32$

$\begin{array}{llll}1(0) & 1(0) & 1(0) & 0,81\end{array}$

$1(0) \quad 1(0) \quad 1(0) \quad 0,67$

$1(0) \quad 1(0) \quad 0,31$

$1(0) \quad 1(0) \quad 0,07$

$1(0) \quad 1(0) \quad 0,38$

$1(0) \quad 1(0) \quad 1(0) \quad 0,81$

$1(0) \quad 1(0) \quad 1(0) \quad 0,35$

$1(0) \quad 1(0) \quad 1(0) \quad 0,37$

$1(0) \quad 1(0) \quad 1(0) \quad 0,83$

$1(0) \quad 1(0) \quad 1(0) \quad 0,11$

Nota: $\mathrm{Me}$ = valor mediano; IQR = rango intercuartílico. Cada puntuación obtenida se basa en una escala Likert 
No se encuentran diferencias significativas por género en las correlaciones realizadas con los diferentes ítems salvo en el ítem 2 "Han copiado mis conversaciones privadas y se las han enviado a otros, para dañarme" $\left(p={ }^{*} 0,02\right)$.

El ítem relacionado con la exclusión de plataformas digitales (ítem $n^{\circ} 3$ ) obtuvo una elevada puntuación debido a que los juegos representan uno de los primeros contactos de los adolescentes con las nuevas tecnologías e internet, esta situación predomina en los cursos más bajos de la ESO (12-13 años), lo que coincide con la media de 13,73 señalada por Ballesteros et al., (2018). El resultado se replica en chats o RRSS (ítem $n^{\circ} 6$ ). A este respecto, el rápido surgimiento de nuevas aplicaciones relacionadas con RRSS puede incrementar la discriminación.

El ítem número 8 sobre las llamadas anónimas tiene una elevada puntuación, convirtiéndose en una de las formas preferidas de ciberacoso por los adolescentes. También el ítem 15 debido al posicionamiento de WhatsApp como uno de los medios preferidos por los ciberacosadores ya en ambos casos realiza las agresiones instantáneamente y puede refugiarse en el anonimato.

Las conductas relacionadas con la suplantación de identidad (ítem $n^{\circ}$ 5) y el bloqueo o cambio de contraseñas en plataformas (ítem $n^{\circ} 12$ ) han tenido una mayor frecuencia en cursos altos de la ESO, $\left(3^{\circ}\right.$ y $\left.4^{\circ}\right)$ ligado a un mayor nivel de conocimientos informáticos y competencias digitales. Finalmente, la mayor audiencia de internet eleva el daño ocasionado por calumnias y difamaciones (ítem $\mathrm{n}^{\circ} 26$ ).

\section{Tabla 4.}

Análisis descriptivo de cada dimensión del cuestionario.

\begin{tabular}{lcccc}
\hline & & \multicolumn{4}{c}{ Género } \\
\hline \multirow{3}{*}{ Dimensiones } & Me (IQR) & Hombre & Mujer & $\boldsymbol{p}$ \\
Cibervictimización visual & $1(0)$ & $1(0)$ & $1(0)$ & 0,91 \\
Exclusión online & $1(0,5)$ & $1(0,5)$ & $1(0,5)$ & 0,89 \\
Suplantación & $1(0)$ & $1(0,0)$ & $1(0)$ & 0,36 \\
Cibervictimización verbal-escrita & $1,08(0,33)$ & $1(0,25)$ & $1,16(0,41)$ & $*_{0,03}$ \\
\hline
\end{tabular}

Nota: $\mathrm{Me}=$ valor mediano; IQR = rango intercuartílico. Cada puntuación obtenida se basa en una escala Likert

Para el análisis descriptivo de las cuatro dimensiones del cuestionario se obtuvieron puntuaciones a partir del valor mediano $(\mathrm{Me})$ de los ítems que conformaba cada dimensión. Los datos son presentados en la Tabla 4 como mediana y rango intercuartílico (IQR).

Únicamente en la cuarta dimensión "Cibervictimización verbal-escrita" se obtienen diferencias significativas por género $(p=* 0,03)$. Los participantes muestran una puntuación mediana $(\mathrm{Me})=1,08(\mathrm{IQR}=0,33)$, observándose resultados más bajos en los hombres, MeHombres=1 $(\mathrm{IQR}=0,25)$ que en mujeres MeMujeres=1,16 (IRQ=0,41). Este resultado contrasta con lo señalado por Alvitez, (2019) sobre la cibervictimización visual como la más asidua, resaltando la verbal-escrita como la que más común.

En relación a la hipótesis planteada, solo se cumple de manera parcial ya que solo se observa una diferencia significativa entre géneros en la cibervictimización verbal-escrita sin encontrar diferencias en los otros tres ámbitos de estudio. 


\section{Tabla 5.}

Correlaciones entre las dimensiones y la variable curso.

\begin{tabular}{lc}
\hline \multicolumn{1}{c}{ Dimensiones } & $\operatorname{Edad} \rho(p)$ \\
\hline Cibervictimización visual & $0,09(0,27)$ \\
Exclusión online & $-0,20\left({ }^{*} 0,01\right)$ \\
Suplantación & $-0,04(0,56)$ \\
Cibervictimización verbal-escrita & $-1,013(0,09)$ \\
\hline
\end{tabular}

Nota: La correlación es significativa en ** $\mathrm{p}<0,01 ; * \mathrm{p}<0,05$. Cada puntuación obtenida en las dimensiones se basa en una escala Likert.

La tabla 5 muestra las correlaciones resultantes entre las dimensiones que configuran el cuestionario de Cibervictimización y los diferentes cursos escolares realizados mediante la prueba de Spearman. La confiabilidad de los datos se calculó utilizando el Alpha de Cronbach para cada una de las dimensiones.

Únicamente la dimensión 2 "Exclusión Online” presenta una correlación débil e inversa ( $\mathrm{p}=$ 0,$\left.20 ; p={ }^{*} 0,01\right)$, que sugiere que a medida que aumenta el rango de edad disminuye la Exclusión Online.

\section{Conclusiones}

El fenómeno del ciberacoso sigue latente en las aulas de educación secundaria obligatoria, siendo necesario un importante trabajo en sinergia familia-escuela de cara a la prevención de este tipo de conductas derivadas de un mal uso de las RRSS. Por ende, se hace fundamental que los adolescentes e incluso estudiantes de primaria se inicien en un trabajo de alfabetización digital, que trabaje aspectos y contenidos de competencia digital para guiarles en el uso de las nuevas tecnologías. Será importante el mantenimiento por parte de las instituciones educativas de los programas de acción preventiva de cara a erradicar el problema. Esto ayudará a las víctimas a empoderarse y poder plantar cara a los ciberacosadores. El control parental desde edades tempranas sobre todo en el uso del teléfono móvil también resulta imprescindible, ya que ha demostrado ser el medio más empleado en este tipo de delitos.

Una de las limitaciones del estudio es que suscribir la muestra al alumnado de educación secundaria obligatoria el colegio Santa Cecilia - Cáceres, ha impedido seleccionar la muestra de acuerdo a procedimientos aleatorios. También el tamaño de la muestra resulta limitado, por lo que no podremos considerarla como representativa y deberemos interpretar los datos obtenidos con cautela. De igual manera, todos los alumnos participantes pertenecen a un mismo centro educativo lo que puede llevar a obtener datos sesgados en la muestra.

Una ampliación de la muestra en estudios futuros ayudaría a conseguir datos más significativos, y con ello, una considerable mejora en la validez externa. Por todo ello, y como futuras líneas de investigación, podría intentarse dar respuesta a los numerosos interrogantes que aún quedan sin clarificar en torno al fenómeno del ciberacoso, como por ejemplo, la influencia del delito sobre el rendimiento académico de los alumnos afectados, o la validez de medidas de prevención instauradas en los centros educativos en torno al ciberacoso. 


\section{Referencias}

Álvarez-García, Dobarro y Núñez, (2014). Validez y fiabilidad del cuestionario de Cibervictimización en estudiantes de Secundaria. Aula abierta (43), 32-38. http://dx.doi.org/10.1016/j.aula.2014.11.001

Amemiya, I., Oliveros, M., \& Barrientos, A. (2009). Factores de riesgo de violencia escolar (bullying) severa en colegios privados de tres zonas de la sierra del Perú. Anales De La Facultad De Medicina, 70(4), 255-258. https://doi.org/10.15381/anales.v70i4.925

Anderson, T., y Kanuka, H. (2002). e-Research: Allyn \& Bacon.

Avilés, J.Ma. (2009). Ciberbullying. Diferencias entre el alumnado de secundaria. Boletín de psicología, 96, 79-96.

Baldry, A. C., Farrington, D. P., \& Sorrentino, A. (2015). “Am I at risk of cyberbullying”? A narrative review and conceptual framework for research on risk of cyberbullying and cybervictimization: The risk and needs assessment approach. Aggression and Violent Behavior, 23, 36-51.

Ballesteros B., Pérez de V., S., Díaz, D., y Toledano, E. (2018). III Estudio sobre acoso escolar y ciberbullying según los afectados: Informe del teléfono. Fundación Mutua Madrileña y Fundación ANAR. Dispononible file:///C:/Users/Juan\%20Carlos/Downloads/4_INFORME_III_ESTUDIO_CIBERBULLYIN G.pdf

Belsey, B. (2005). Cyberbullying: An emerging threat to the always of generation. http://www.cyberbullying.ca

Blanco, S., González, C. y Velasco, J. (2020). Nuevas herramientas para viejos problemas: acoso y ciberacoso a través de un análisis de sentencias. Tecnología, Ciencia y Educación, 15, 51-80. https://doi.org/10.51302/tce.2020.367

De la Plaza, M. y González, H. (2019). El acoso escolar: factores de riesgo, protección y consecuencias en víctimas y acosadores. Revista de Victimología, (9), 99-13. https://doi.org/10.12827/RVJV.9.01

Del Rey, R., Casas J.A. y Ortega R. (2018). El programa ConRed, una práctica basada en la evidencia. Revista Comunicar. Revista científica de Educomunicación, v.XX (39), 129138.

Díaz-Aguado, M.J. (2005). La violencia entre iguales en la adolescencia y su prevención desde la escuela [Peer violence among adolescents and its prevention from school]. Psicothema, 17, 549-558.

Espelage, D. L. \& Swearer, S. M. (2003). Research on school bullying and victimization: what have we learned and where do we go from here? School Psychology Review, 32 (3), pp. 365-383

Fernández, C. M. G., Félix, E. M. R., \& Ruiz, R. O. (2015). Explicative factors of face-to-face harassment and cyberbullying in a sample of primary students. Psicothema, 27(4), 347-353.

Flores J. (2008). Grooming, acoso a menores en la Red. Disponible en: http://www.pantallasamigas.net/ciberbullyingguia-rapida/

Garaigordobil, M., y Oñederra, J.A. (2010). La violencia entre iguales: Revisión teórica y estrategias de intervención. Madrid: Pirámide. 
García, A., y Jiménez, A. (2010). Estudio exploratorio y descriptivo del cyberbullying en escolares de secundaria. Revista Qurriculum, pp155-164. http://riull.ull.es/xmlui/handle/915/13349

Giménez Gualdo, A., Arnaiz, P. y Maquilón, J. J. (2013). Cyberbullying, la nueva cara de la violencia escolar. Académica Española.

Hernández, T. \& O'Reilly, M. B. (2015). Investigación sobre la delincuencia y el bullying escolar en España. Teoría, evolución y tendencias. Valencia: Tirant Humanidades.

IAB Spain (2017). Estudio anual redes sociales 2017. https://iabspain.es/estudio/estudio- anualde-redes-sociales-2017/

Kowalski, R., Limber, S., Agatston, P. (2010). Cyberbullying: El acoso escolar en la era digital. Bilbao, Editorial Desclée de Brouwer.

López, M.C. y Sánchez, C. (2019). La interacción y convivencia digital de los estudiantes en las redes sociales. Revista de Educación Inclusiva, 12(2), 114-130.

Martín, A. R., Cabré, R. B., \& Neri, E. M. (2019). Ciberacoso y ansiedad social en adolescentes: una revisión sistemática. Revista de Psicología Clínica con niños y adolescentes, 6(1), 9-15.

Salkind, N. J. (1999). Métodos de investigación: Pearson Educación.

Sapountzi, A., \& Psannis, K. E. (2018). Social networking data analysis tools \& challenges. Future Generation Computer Systems, 86, 893-913. doi:10.1016/j.future.2016.10.019

UNICEF. (2019). Día Internet Segura: al menos 2 estudiantes en cada aula sufren acoso o violencia en España. https://www.unicef.es/noticia/dia-internet-segura-al-menos-2estudiantes-en-cada-aulasufren-acoso-o-violencia-en-espana

Wolke, D., \& Lereya, S. T. (2015). Long-term effects of bullying. Archives of Disease in Childhood, 100, 879-885. https://doi.org/10.1136/archdischild-2014-306667.

Willard, N. E. (2006). Cyberbullying and cyberthreats: Responding to the challenge of online social aggression, threats and distress. Eugene: Center for Safe and Responsible Internet Use. 